\title{
A Study of Prognosis, Outcome, and Changing Tendency of Hospitalized AMI Patients in Beijing Third-Grade A-Level Traditional Chinese Medicine Hospitals from 1999 to 2008
}

\author{
Ju-Ju Shang, ${ }^{1}$ Hui Shi, ${ }^{1}$ Qi Zhou, ${ }^{1}$ Wei Gao, ${ }^{1}$ and Hong-xu Liu' \\ ${ }^{1}$ Beijing Traditional Chinese Medicine Hospital, Capital Medical University, Dongcheng, Beijing 100010, China \\ ${ }^{2}$ Cardiovascular Department, Beijing Traditional Chinese Medicine Hospital, Capital Medical University, Beijing 100010, China
}

Correspondence should be addressed to Hong-xu Liu, lhx_@263.net

Received 23 November 2011; Accepted 12 January 2012

Academic Editor: Myeong Soo Lee

Copyright ( $\odot 2012$ Ju-Ju Shang et al. This is an open access article distributed under the Creative Commons Attribution License, which permits unrestricted use, distribution, and reproduction in any medium, provided the original work is properly cited.

Objectives. To survey and analyse the prognosis, outcome, and changing tendency of the Acute Myocardial Infarction (AMI) patients in Beijing third-grade A-level Traditional Chinese Medicine (TCM) hospitals. Methods. We collected the clinical datum of hospitalized AMI patients in Beijing 6 TCM hospitals from January 1999 to December 2008 and then analysed the clinical datum. Results. (1) The mean age of patients had showed a slowly rising tendency during this ten years. The patients who had previous history of cerebrovascular diseasea and multiple comorbidities had increased year by year. (2) The rate of reperfusion therapy, revascularization and standardized using of drug, and usage of TCM of AMI patients presented a significant increasing tendency in these hospitals. (3) The proportion of AMI patients combined with cardiac arrhythmia and heart failure had decreased significantly. (4) The AMI mortality presented a decreasing tendency in 10 years. Conclusions. The AMI patients in Beijing TCM hospitals had their own unique clinical features, and it can improve their prognosis by combined therapy of Western Medicine and Traditional Chinese Medicine.

\section{Objectives}

Cross-sectional registry was used to collect the hospitalized AMI patients' clinical datum in third-grade A-level TCM hospitals from 1999 to 2008 in Beijing, so as to dynamically analyse their clinical characteristics in recent 10 years and provide preliminary epidemiological datum of standardized treatment in TCM hospitals.

\section{Participants}

2.1. Source of Cases. The subjects we enrolled were hospitalized patients suffering from AMI in Beijing Third-grade A-Level TCM hospitals from January 1, 1999 to December 31,2008 . We totaly recovered 2056 case report form (CRF) handbooks, and by screening there were 2053 within registered. See Table 1.
2.2. Inclusion Criteria. (1) All participants in the study hospital's records and statistics room in the computer management system of the international classification of diseases (ICD) were coded for AMI; (2). All these patients were diagnosed as AMI by reference of the Chinese society of Cardiology, Chinese Journal of cardiovascular medicine, Editorial Committee of Chinese Circulation Journal jointly developed the Guidelines for the Diagnosis and Treatment of Acute Myocardial Infarction (2001) [1] (the following referred to as the Guideline). (3) The information of research-related syndromes of Chinese medicine were filled or by adding completely then could be used. Conforming to the above three criteria can be inclused into the survey.

2.3. Exclusion Criteria. (1) Old AMI patients. (2) Patients' TCM syndrome information was seriously uncomplete and unable to complement. In accordance with neither of the two criteria, it would be been exclused. 
TABLE 1: List of hospitals participating in the survey.

\begin{tabular}{lcc}
\hline Hospital name & Cases & Proportion (\%) \\
\hline Beijing TCM Hospital & 479 & 23.33 \\
Xi Yuan TCM Hospital & 584 & 28.45 \\
Dong Fang TCM Hospital & 456 & 22.21 \\
Guang, anmen TCM Hospital & 248 & 12.08 \\
Dongzhimen TCM Hospital & 204 & 9.94 \\
Wangjing TCM Hospital & 82 & 3.99 \\
\hline
\end{tabular}

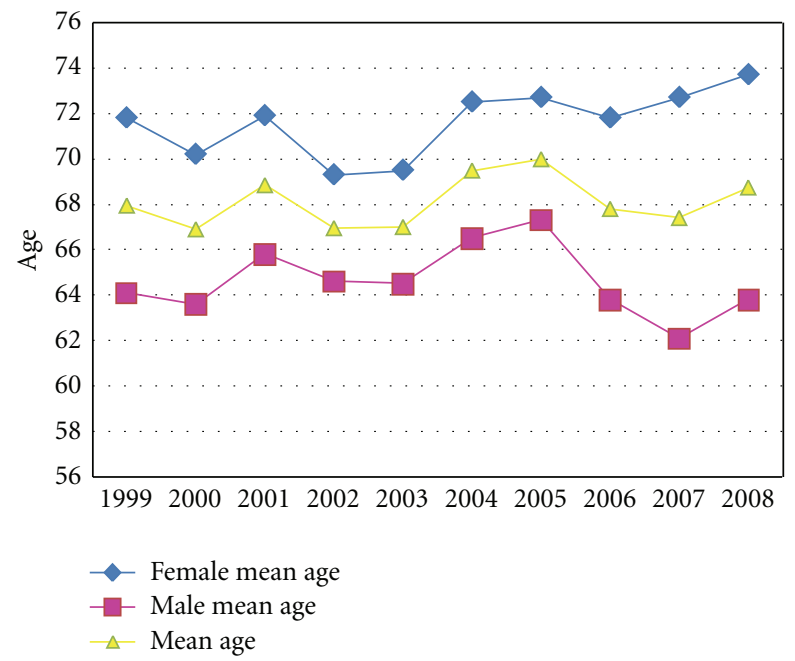

Figure 1: The age change trend of AMI patients in third-grade Alevel TCM hospitals in Beijing from 1999 to 2008.

\section{Methods}

First of all the Capital Research Foundation for Medical Development group designed the CRF handbook which included the general characteristics, hospitalized therapeutic conditions and the prognosis, and outcome of AMI patients. Then we gave the CRF to the doctors of cardiovascular department of each hospital. In every hospital, elected cases were filled according to the actual situation by the first doctor who had been trained. About every six months, all the cases of cooperate units were summarized to the cardiovascular department of Beijing TCM Hospital, then the task group inspected the datum and eliminated invalid datum.

Next the datum was entered to the survey database which was established based on ACESS 2000 by China Department of Epidemiology, Capital Medical University Beijing Anzhen Hospital. The datum was entered by the trained persons. We used double datum entry, consolidated datum, and then checked error by the logic difference method. Statistical software SPSS15.0 (SPSS Base15.0S, PN: 32119001, SN: 5045602, L20080005) was used in descriptive analysis for general datum, measurement datum was analyzed by $F$ test and $Q$ test, and two sets of measurement datum were analyzed by $T$ test; categorical data was analyzed by $X^{2}$ test.

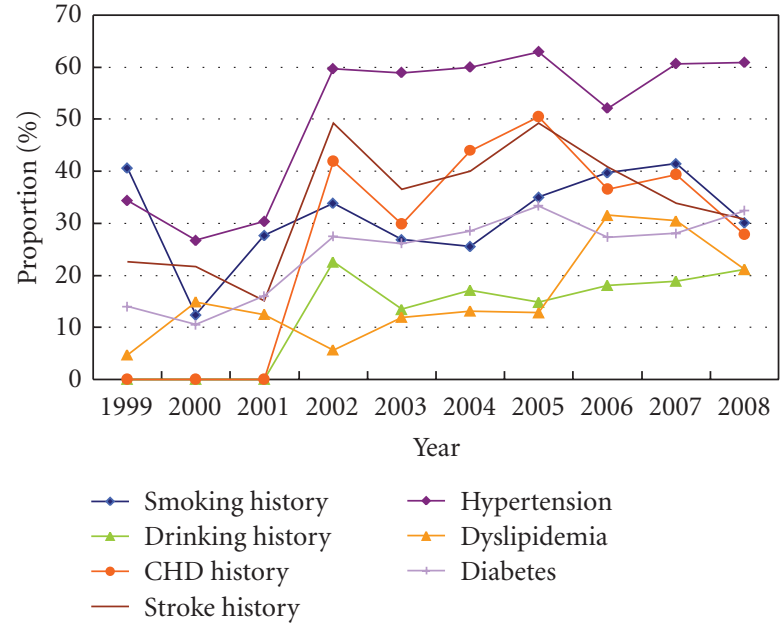

FIgURE 2: The risk factors change trend of AMI patients in thirdgrade A-level TCM hospitals in Beijing from 1999 to 2008.

\section{Results}

\subsection{General Characteristics}

4.1.1. Age. A total of 2053 AMI patients were entered, of these, 2015 cases had clearly recorded the age of onset, the minimum age was 18 years old, the maximum age was 105 years old, and the mean age was $67.37 \pm 12.10$ years old. The average onset age showed a slowly rising tendency from 1999 to 2008 . 1316 cases were male patients, 737 cases were female patients, and the gender ratio was $1.8: 1$. For the changing tendency of AMI patients' see Figure 1.

4.1.2. The History and Risk Factors. There were total 680 patients with a clear history of smoking and 299 cases with a history of clear drinking. Patients with a history of smoking drinking in the proportion of overall show a slow ascending tendency. In 638 cases with clear history of coronary heart disease, the proportion had decreased. 695 patients had a clear stroke history, the proportion had gone up. 1130 patients combined with hypertension, and 438 patients had dyslipidemia, 542 patients combined with diabetes mellitus; the proportion of those patients to the overall was in a upward trend from 1999 to 2008 (see Figure 2).

4.1.3. AMI Status. The total anterior wall myocardial infarction (MI) cases was 1324, and the proportion had no obvious change; the rear wall MI cases were 1147, right ventricular MI cases were 170, and the proportion of inferior wall and right ventricular MI decreased slowly. Clear ST segment elevation cases were 1287; clear Q wave MI cases were 1231; 1616 cases were the first MI, 243 cases were second MI, 16 cases were third MI, and 178 cases were unspecified (see Figure 3 ).

A total of 656 patients underwent coronary angiography, accounted for 31.95 percentage of the observed cases; patients undergoing coronary angiography increased year by year from 2001 to 2008 . 


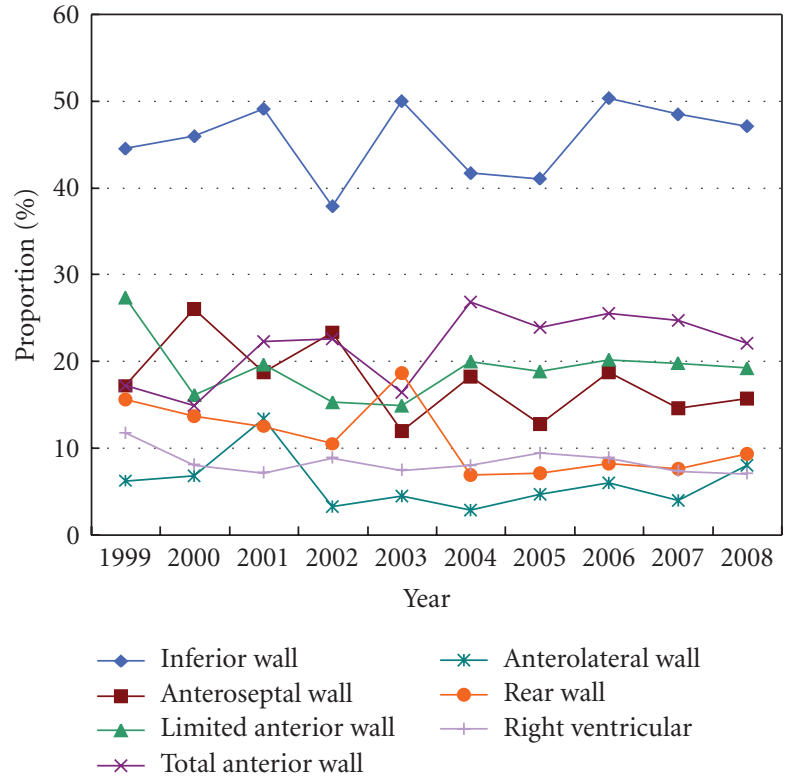

FIGURE 3: The proportion of MI location of hospitalized AMI patients from 1999 to 2008 .

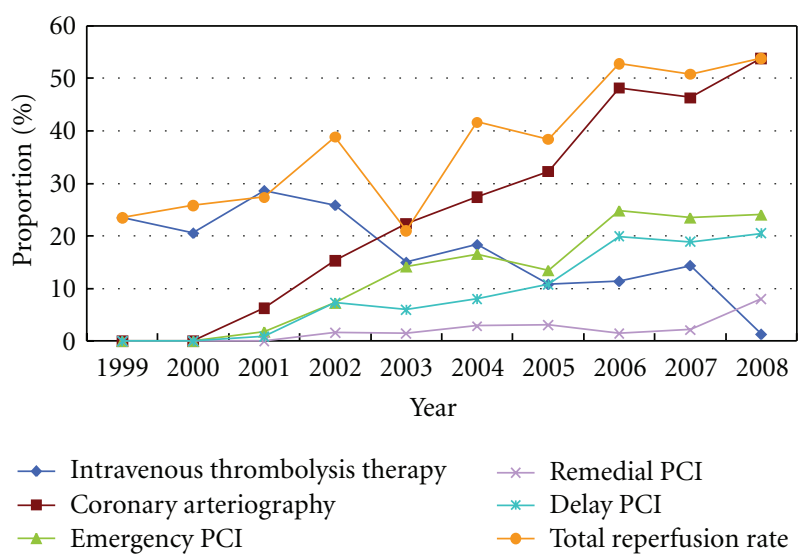

FIGURE 4: the reperfusion therapy conditions of AMI patients in third-grade A-level TCM hospitals in Beijing from 1999 to 2008.

\subsection{Hospitalized Therapeutic Conditions}

4.2.1. Reperfusion Therapy. In 2003, the usage rate of reperfusion therapy was the lowest $(20.97 \%)$, while in 2008 , it had gone up to $53.80 \%$, which presented an upward tendency from 1999 to 2008. The usage rate of intravenous thrombolysis therapy was the lowest in 2008 (1.28\%), while in 2008 , it was the highest up to $28.57 \%$, which presented a downward tendency from 1999 to 2008.

There was no emergency PCI patient in 1999 and 2000. The rate of underwent emergency PCI was the lowest in 2008 $(1.79 \%)$, while in 2006 , it was the highest up to $24.82 \%$, which presented an upward tendency from 1999 to 2008. The rate of underwent early reperfusion was the lowest in 1999 (23.44\%), while in 2007 , it was the highest up to $37.80 \%$ which presented an upward tendency from 1999 to 2008.
There was no patients undergoing CABG in 2000, 1999, 2007, and 2008, CABG patients have the highest proportion (1.61\%) in 2002, and from 1999 to 2008 there had been no obvious regularity. There was no patients undergoing remedial PCI in 1999 and 2001. The remedial PCI patients covered the lowest proportion in 2003 as $1.49 \%$ and the highest proportion in 2008 as $8.01 \%$, which presented an ascendant tendency from 1999 to 2008 . There was no patient undergoing selective PCI in 1999 and 2000; the selective PCI patients covered the lowest proportion as $0.89 \%$ in 2001 and the highest as $20.51 \%$ in 2008 , which also presented an ascendant tendency from 1999 to 2008. Specific reperfusion form is shown in Figure 2.

4.2.2. Oral Drug Therapy. Western medicine intervention: the usage rate of drugs followed the Guideline recommendations from 1999 to 2008 which were the following: aspirin was $86.64 \%$, clopidogrel was $37.91 \%$, nitrate was $88.91 \%$, beta blockers were $67.84 \%$, angiotensin-converting enzyme inhibitors (ACEIs) and angiotensin receptor blocker (ARB) together were $77.75 \%$, low molecular weight heparin was $82.38 \%$, and adjustable lipid drug was $54.37 \%$ see Figure 3.

The usage rate of other drugs: from 1999 to 2008 the usage rate of these drugs which were not recommended by the Guideline was the following: unfractionated heparin was $14.4 \%$, antiarrhythmic drug was $16.1 \%$, CCB was $23.4 \%$, diuretic was $39.9 \%$, digitalis cardiac drug was $16 \%$, GIK was 20.4\%. See Figures 4 and 6.

4.2.3. The Intervention's Conditions of Chinese Medicine. From 1999 to 2008, within 2053 patients, 1851 patients have used intravenous preparation of Chinese medicine in TCM hosptitals, and the total usage rate was $90.2 \%$, which presented a tendency of escalation. 400 patients used oral proprietary Chinese medicines, and the general usage rate was $19.5 \%$, which presented a decline in general from 1999 to 2008. 1056 patients using oral decoction, the total usage rate was $51.4 \%$, and the usage rate of decoction of Chinese medicine was increasing in general from 1999 to 2008. The changing tendency of Chinese medicine intervention was shown in Figures 5 and 7.

4.3. Complications. Arrhythmia, heart failure, and cardiogenic shock are the common clinical complications of AMI patients, and are also the major cause of death. Based on the datum of 10 years in Beijing third-grade A-level TCM hospitals, the proportion of patients complicated by arrhythmia had dropped from $42.91 \%$ in 1999 to $24.04 \%$ in 2008, presented a fluctuating downward tendency; the proportion of patients complicated by heart failure had dropped from $60.16 \%$ in 1999 to $41.03 \%$ in 2008 , and presented a significant downward tendency; patients complicated by cardiogenic shock had no obvious changes in the proportion.

4.4. Mortality. This survey showed that the mortality of AMI patients presented a fluctuating tendency of decline from 1999 to 2008 in Beijing Third-grade A-Level TCM hospitals, the main death due to cardiogenic death. With the 


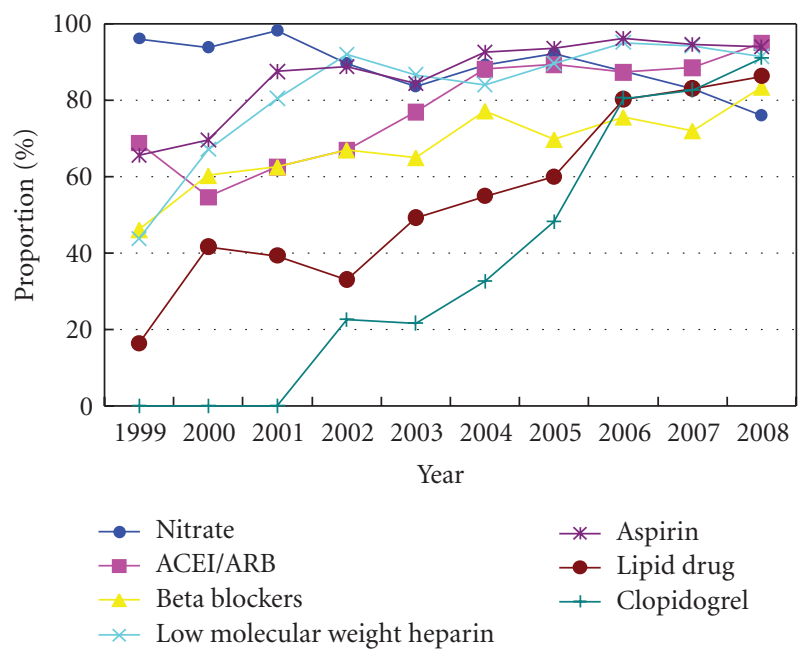

FIgURE 5: The status of oral drug usage followed the Guideline recommended to AMI patients in Third-grade A-Level TCM hospitals in Beijing from 1999 to 2008.

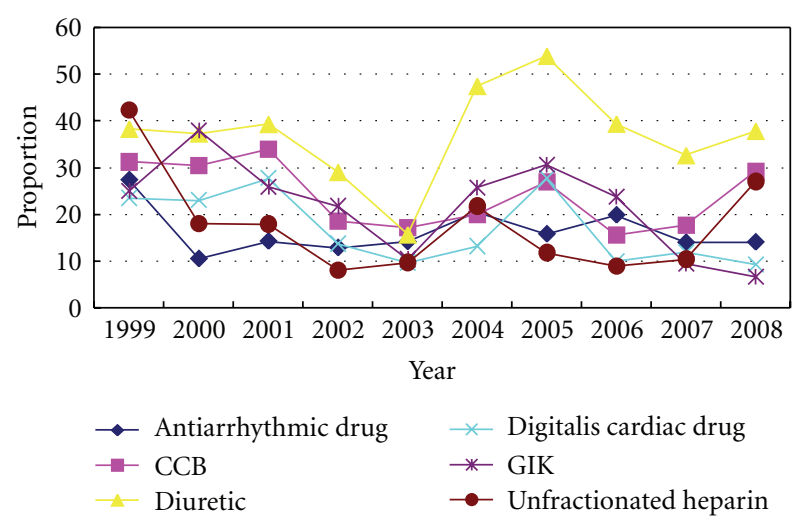

Figure 6: The rate of oral drugs which were not recommended by the Guideline to AMI patients in Third-grade A-Level TCM hospitals in Beijing from 1999 to 2008.

increase of age, AMI's mortality gradually increased, and the hospitalized mortality of patients older than 75 years old was up to $22.91 \%$.

Female AMI patients' hospitalized mortality was higher than that of male, which was $19.15 \%$.

The survey also showed that the mortality of patients accompanied by arrhythmia, heart failure was significantly higher than in those without the complications $(22.5 \%$ versus $8.8 \%, 37.8 \%$ versus $4.4 \%$ ), and especially patients complicated with cardiogenic shock had a high mortality which was upto $56.9 \%$.

\section{Discussion}

AMI is a serious cardiovascular disease which is hazard to human health and is the leading cause of death worldwide. In recent years, with the standardization of early reperfusion and drug treatment AMI mortality declined but it is still a high-mortality disease.

5.1. Clinical Characteristics. This survey presented that the average age of AMI patients was 67.37 years old, the patients older than 65 years old account for $64.40 \%$ of all observed cases, and the male to female ratio was $1.8: 1$.

A 26-year follow-up survey showed that the incidence of AMI of population aged 35 to 84 years old, for males was $71 \%$, for women, was $22 \%$. For the group from 55 to 64 years old, the incidence for male and female was $91 \%$ and $25 \%$, it were $119 \%$ ond $51 \%$ in the $65 \sim 74$ years old group and; was $168 \%$ and $90 \%$ in the $75 \sim 84$ age group [2].

Compared with AMI patients on the study which was also sponsored by our study group to survey the therapeutic situations of TCM hospitals and Western medicine hospitals in Beijing in 2005 [3], we found that AMI patients in thridgrade A-level TCM hospitals in Beijing area tended to be older, and the female proportion was much higher in TCM hospitals than that in western medicine hospitals.

5.2. Therapeutic Conditions. Reperfusion therapy has become the most important means of treatment on AMI. Based on the datum of 10 years, the proportion of reperfusion therapy showed rising, the proportion of intravenous thrombolytic treatment had descended, all these presented the whole reperfusion technology, and levels rose apparently in TCM hospitals in Beijing.

The usage rate of drugs recommended by Guideline presented obviously increased, while the usage rate these drugs not recommended by Guideline presented a fluctuated decline, which prompted the gap between Beijing Third-grade A-level TCM hospitals and the Guideline demand narrowed gradually.

In the development of China Traditional Chinese medicine had been playing an important role in preventing and treating all kinds of diseases. In the treatment of AMI, Chinese medicine also plays its role. This survey showed that Chinese medicine intravenous preparations currently have been widely used in clinically; this may be related to their convenient usage and the effective component being relatively single. Other several studies of our study group all show that Chinese medicine intravenous preparations can lower the mortality [3-6].

5.3. Complications. Arrhythmia, heart failure, and cardiogenic shock are the common clinical complications of AMI patients and are also the major cause of death. The datum showed the proportion of patients complicated by arrhythmia and heart failure presented a significant downward tendency during this 10 years in Beijing thirdgrade A-level TCM hospitals. All these showed that TCM hospitals in Beijing area have made unceasing progress in the treatment concept, treatment means, and so on, with the early reperfusion level rising ceaselessly and Chinese medicine vein preparation widely used clinically in AMI patients, patients can receive timely and effective treatment, and hospitalized complication will decrease gradually. 


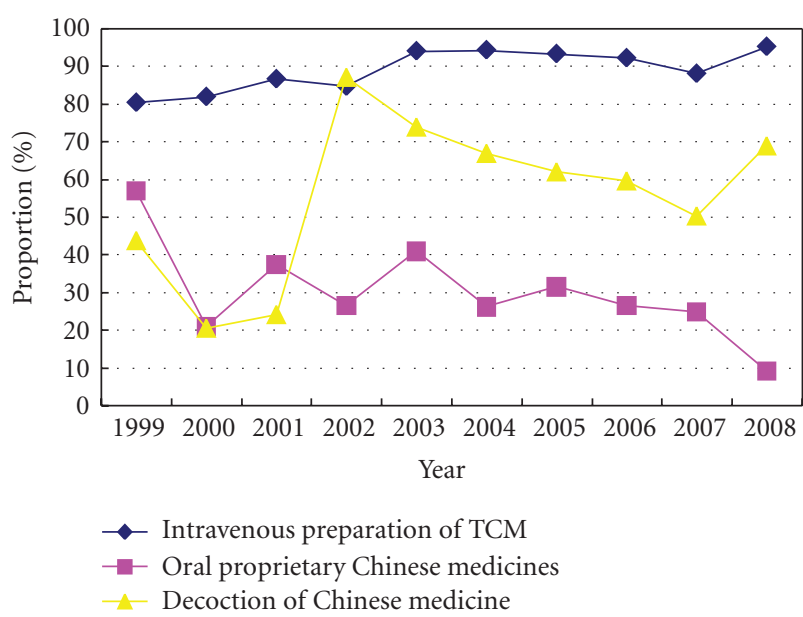

FIgURe 7: Chinese medicine intervention in AMI patients in Beijing Third-grade A-Level hospitals from 1999 to 2008.

5.4. Mortality. The mortality of AMI correlates to age, gender, and complications. Our survey showed that AMI patients who had a history of stroke and coronary heart disease, combined with hypertension, diabetes mellitus patients and many hospitalized history had a relatively higher mortality, considering related to the factors as combined with more disease, relatively complex and worse vascular condition.

Arrhythmia, heart failure, and cardiogenic shock are common complications in patients with AMI; AMI complications and mortality are also highly correlated. The survey also showed that the mortality of patients accompanied by arrhythmia, heart failure was significantly higher than in those without the complications, and especially patients complicated with cardiogenic shock had a high mortality which was up to $56.9 \%$.

\section{Conclusions}

Through this survey, we found patients who suffered from AMI in TCM hospitals in Beijing area were mainly old patients and the female patients were more than those in Western Medicine hospitals, and they had more complications. Our other studies have shown that [7-9] elder AMI patients either in clinical features, combined with risk factors, treatment, or on long- or short-term prognosis were significantly different from young patients, and especially for older female patients, it was different from male patients.

Traditional Chinese medicine had been playing an important role in preventing and treating all kinds of diseases for Chinese people. In the treatment of AMI, Chinese medicine also plays its role. Integrative medicine treatment, combining TCM and conventional medicine, has been the most representative characteristic for AMI. However, the potential benefit of integrative medicine therapy in improving AMI prognosis is still under studying. Our study showed that, by combined therapy of Western Medicine and Traditional Chinese Medicine, the mortality of AMI patients in Beijing TCM Hospitals presented a decreasing tendency, which presented that the integrative medicine might have potential benefit for AMI patients.

\section{Sources of Funding}

This project was financially supported by Capital Research Foundation for Medical Development, Beijing Medicine and Health Science Collaborative Research Project, no. 2003I4/SF-2009-III-20.

\section{Acknowledgments}

The authors are indebted to all the staff members of all the participating hospitals and centers for their outstanding efforts: Mei JIN, Hong-yi Jing, Xiao-lei LAI, Tong TONG, Jia-xing CHEN, Beijing Hospital of Traditional Chinese Medicine, Capital Medical University; Da-zhuo SHI, Yan LEI, Yang MIAO, Xiao-sha WANG, Xiyuan Hospital, China Academy of Chinese Medical Sciences; Qian LIN, Yanming HUO, Yi-bing NONG, Dongfang TCM Hospital; Wei-xing LU, Yu-qing LIU, Yu-feng LI, Dongzhimen TCM Hospital; Yuan-hui HU, Yu-tao FANG, Xiu-yang SHANG, GuangAnMen TCM Hospital; references Hasai D Begar S, Wallentin L, Barakat K, Wilkinson P, Deaner A, Sakai K, Nakagawa Y, Kimura T, and Milner KA, Vaccarino V, Arnold AL et al.

\section{References}

[1] Chinese society of Cardiology, "AMI guidelines for diagnosis and treatment," Chinese Journal of Cardiology, vol. 29, no. 12, pp. 710-725, 2001.

[2] D. Hasdai, S. Behar, L. Wallentin et al., "A prospective survey of the characteristics, treatments and outcomes of patients with acute coronary syndromes in Europe and the Mediterranean basin: the euro heart survey of acute coronary syndromes (Euro Heart Survey ACS)," European Heart Journal, vol. 23, no. 15, pp. 1190-1201, 2002.

[3] W. Gao, H.-X. Liu, J.-J. Shang, and Q. Zhou, "Comparative study of clinical characteristics in patients with AMI in thirdgrade A-Level TCM and western medicine hospitals in Beijing," in Proceedings of the Conference Papers Selection of China Association of TCM Heart Disease Branch of the 10th National Academic Annual Conference and Traditional Chinese Medicine Institute of the 2nd Academic Conference of Jilin Province, 2008.

[4] S.-R. Wang, H.-X. Liu, D. Zhao et al., "Study on the therapeutic status of 1242 hospitalized acute myocardial infarction patients in Beijing," Chinese Journal of Epidemiology, vol. 27, no. 11, pp. 79-83, 2006.

[5] H.-X. Liu and Q. Zhou, "The correlated analysis of mortality in AMI patients in Beijing third-grade A-Level TCM hospitals," in Proceedings of the 9th National Symposium on TCM Disease Sselection, 2007.

[6] L. Zheng, H.-X. Liu, J.-J. Shang et al., "Chinese medicine intravenous agents as adjunctive therapy for AMI on mortality and recanalization rate of meta analysis," Journal of Emergency in Traditional Chinese Medicine, vol. 16, no. 7, pp. 859-862, 2007.

[7] K. Barakat, P. Wilkinson, A. Deaner, D. Fluck, K. Ranjadayalan, and A. Timmis, "How should age affect management of acute 
myocardial infarction? A prospective cohort study," The Lancet, vol. 353, no. 9157, pp. 955-959, 1999.

[8] K. Sakai, Y. Nakagawa, T. Kimura et al., "Comparison of results of coronary angioplasty for acute myocardial infarction in patients $\geq 75$ years of age versus patients $<75$ years of age," American Journal of Cardiology, vol. 89, no. 7, pp. 797-800, 2002.

[9] K. A. Milner, V. Vaccarino, A. L. Arnold, M. Funk, and R. J. Goldberg, "Gender and age differences in chief complaints of acute myocardial infarction," American Journal of Cardiology, vol. 93, no. 5, pp. 606-608, 2004. 


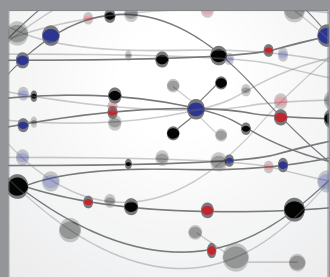

The Scientific World Journal
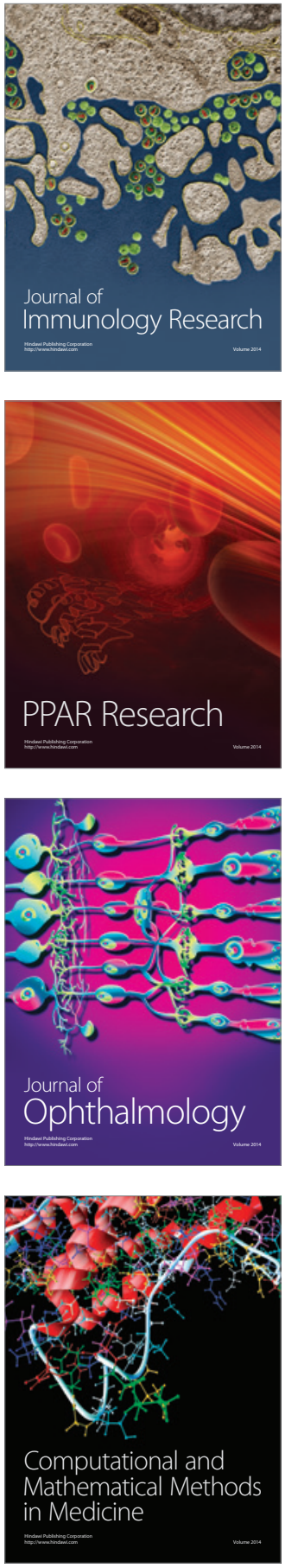

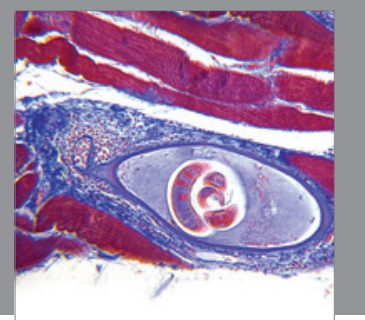

Gastroenterology

Research and Practice
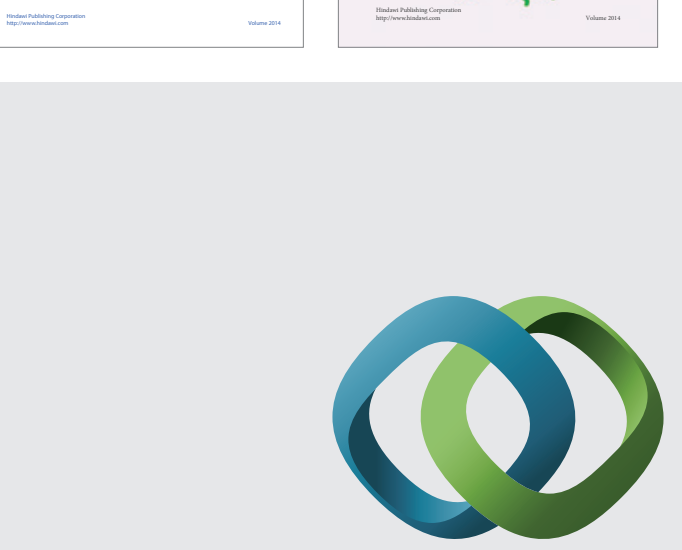

\section{Hindawi}

Submit your manuscripts at

http://www.hindawi.com
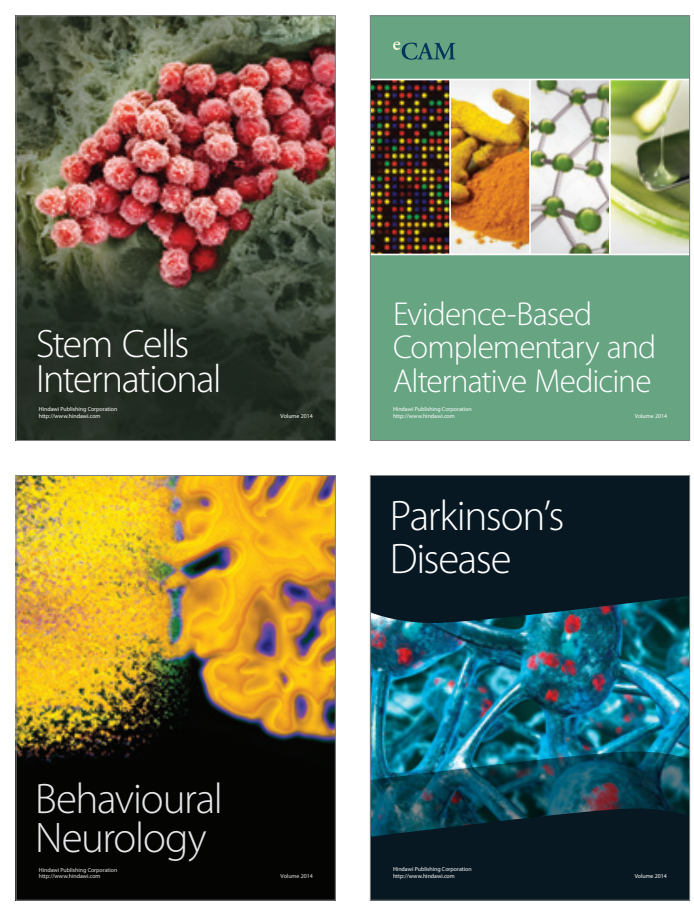

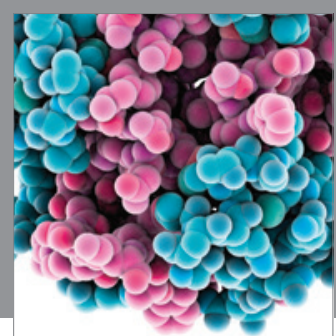

Journal of
Diabetes Research

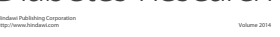

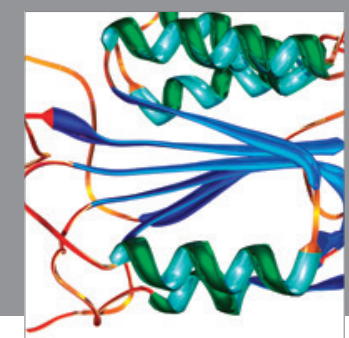

Disease Markers
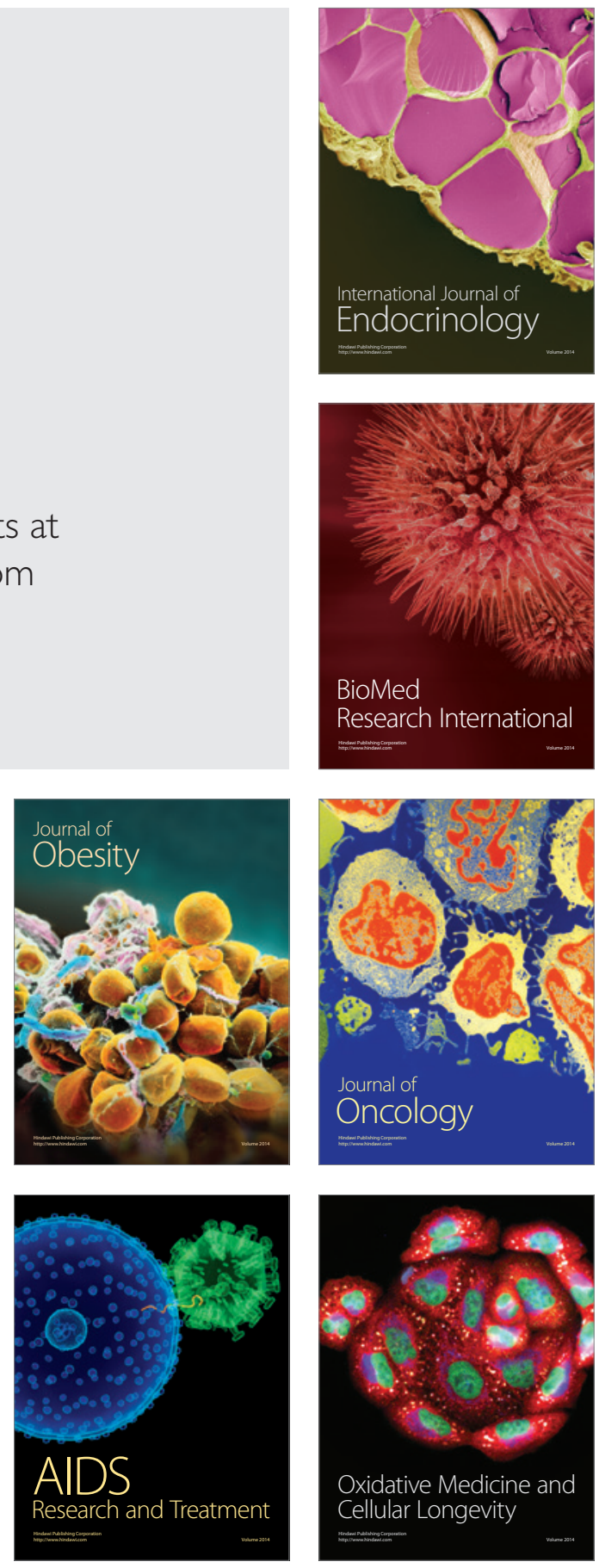